\title{
ORIENTATION-REVERSING PERIODIC PL MAPS OF LENS SPACES
}

\author{
PAIK KEE KIM
}

\begin{abstract}
We complete the classification of all orientation-reversing PL maps of period $4 k(k>1)$ on lens spaces. All $Z_{4}$-actions on the projective 3-space are also classified.
\end{abstract}

1. Introduction. The 3-sphere and the projective 3-space are the only 3-dimensional lens spaces $L(p, q)$ which admit orientation-reversing PL maps of period $n>4$ [4]. In [4] all orientation-reversing PL maps of period $4 k$ on the 3-sphere $S^{3}$ are classified for all $k$. In this paper we show:

THEOREM A. The projective 3-space $P^{3}$ admits a unique orientation-reversing $P L$ map of period $4 k$ for each $k$, up to conjugation.

All orientation-reversing PL involutions of lens spaces [6], [8] and all PL involutions of $P^{3}$ [2], [6] are known. As consequences of Theorem $A$ and [3], we have

THEOREM B. The projective 3-space $P^{3}$ admits exactly four distinct $Z_{4}$-actions $(P L)$, up to conjugation.

The cyclic group generated by $h$ shall be denoted by $\langle h\rangle$. Two actions of $\langle h\rangle$ and $\left\langle h^{\prime}\right\rangle$ on a space $M$ are said to be conjugate if there exists a homeomorphism $t$ of $M$ such that $\left\langle t h t^{-1}\right\rangle=\left\langle h^{\prime}\right\rangle$. We shall denote the fixed-point set of $h$ by $\operatorname{Fix}(h)$.

We consider $S^{3}$ as a subset of $C^{2}$, defined by $\left\{\left(z_{1}, z_{2}\right) \in C^{2} \mid z_{1} \bar{z}_{1}+z_{2} \bar{z}_{2}=\right.$ 1). Define an orientation-reversing homeomorphism $\lambda^{\prime}$ of $S^{3}$ by $\lambda^{\prime}\left(z_{1}, z_{2}\right)=$ $\left(\omega z_{1}, \bar{z}_{2}\right)$, where $\omega=e^{2 \pi i / n}$ and $n$ is even. Since $\lambda^{\prime}$ commutes with the antipodal map of $S^{3}$, there exists an obvious orientation-reversing map $\lambda$ of period $n$ on $P^{3}$ induced by $\lambda^{\prime}$.

REMARK. Let $f$ be an orientation-preserving map of period $n$ on $S^{3}$ and $\operatorname{Fix}(f) \neq \varnothing$. Then $\operatorname{Fix}(f)$ is a simple closed curve. A well-known conjecture, due to P. A. Smith, asserts that $\operatorname{Fix}(f)$ is unknotted for all $n$ (see [1]). It follows from a result of $F$. Waldhausen [12] that the conjecture is true for even period $n$. Let $h$ be an orientation-reversing map of period $n>2$ on $P^{3}$. Then it follows from the Lefschetz fixed-point theorem that $\operatorname{Fix}(h) \neq \varnothing$. Note

Received by the editors November 17, 1975, and, in revised form, September 29, 1976.

AMS (MOS) subject classifications (1970). Primary 55C35, 57A10; Secondary 57E25.

Key words and phrases. Cyclic group action, periodic homeomorphism, fixed-point set, lens space. 
that $h^{2}$ is orientation-preserving and $n$ is even. Assuming the Smith conjecture, one can prove that $\pi_{1}\left(P^{3}-\operatorname{Fix}\left(h^{2}\right)\right)$ is abelian. The following theorem classifies all orientation-reversing periodic maps of $P^{3}$ modulo the Smith conjecture.

THEOREM C. Let $h$ be an orientation-reversing $P L$ map of the projective 3-space $P^{3}$ with period $n>2$. If $\pi_{1}\left(P^{3}-\operatorname{Fix}\left(h^{2}\right)\right)$ is abelian, then $h$ is conjugate to $\lambda$.

Let $h$ be a periodic map of a space $M$. Then there exists a homeomorphism $q$ of $M /\left\langle h^{k}\right\rangle$, uniquely determined by $h$, such that $q g=g h$, where $g$ : $M \rightarrow M /\left\langle h^{k}\right\rangle$ is the orbit map. We call $q$ the map of $M /\left\langle h^{k}\right\rangle$ induced by $h$. We shall denote the closed unit interval and the $n$-sphere by $I$ and $S^{n}$, respectively. In this paper all spaces and maps will be in the PL category.

2. Equivariant product structure. Let $A$ be the annulus $S^{1} \times I$ and let $h$ be a free involution on $A \times I$ such that $h(A \times\{i\})=A \times\{i\}(i=0,1)$. The following lemma is essentially a special case of a result of Waldhausen [11].

Lemma 2.1. There exists an isotopy of $A \times I$, keeping $A \times\{i\}$ invariant, after which a product structure on $A \times I$ and an involution $g$ on $A$ are obtained so that $h(x, t)=(g(x), t)$ for $(x, t) \in A \times I$. The isotopy can be chosen constant on $A \times\{0\}$.

Suppose that $A \times I$ is a regular neighborhood of an invariant simple closed curve $J$, or equivalently $\pi_{1}(A \times I-J)=Z \oplus Z$. The following lemma further claims that the isotopy in Lemma 2.1 can be chosen, after which $J=S^{1} \times\{1 / 2\} \times\{1 / 2\}$.

LEMMA 2.2. There exists an isotopy of $A \times I$, keeping $A \times\{i\}$ invariant, after which a product structure on $A \times I$ and an involution $g$ on $A$ are obtained so that $h(x, t)=(g(x), t)$ for $(x, t) \in A \times I$ and $J=S^{1} \times\{1 / 2\} \times\{1 / 2\}$.

Proof. We will show that there exists an invariant annulus $H$ properly embedded in $A \times I$ such that $\partial H$ meets each $S^{1} \times\{i\} \times(0,1)(i=1,0)$ in a simple closed curve and $J \subset$ Int $H$. Then $H$ separates $A \times I$ into two invariant components, each of which is homeomorphic to $S^{1} \times I \times I$. Define a product structure on each component $S^{1} \times I \times I$ as in Lemma 2.1. We may assume that $J=S^{1} \times\{1 / 2\} \subset S^{1} \times I \approx H$. Now repairing the cut along $H$ defines the required product structure of $A \times I$.

Let $M=A \times I /\langle h\rangle$ and $g: A \times I \rightarrow M$ be the orbit map. Then $M$ is a homeomorphic to $c \times I$, where $c$ is either an annulus or a möbius band. It is not difficult to see that $M$ is a regular neighborhood of $g(J)$. Therefore, there exists a properly embedded surface $H^{\prime}$ in $M$ such that each component of $\partial M-(g(A \times\{0,1\}))$ meets $H^{\prime}$ in a simple closed curve, where $H^{\prime}$ is a regular neighborhood of $g(J)$ in $H^{\prime}$ (see Lemma 2.1). Then $H=g^{-1}\left(H^{\prime}\right)$ is an annulus as desired. 


\section{Proof of Theorem A.}

(3.1) Consider an orientation-reversing map $h$ of period $4 k$ on $P^{3}$. It follows from the Lefschetz fixed-point theorem that $\operatorname{Fix}(h) \neq \varnothing$. Since $h^{2 k}$ is an involution of $P^{3}, \operatorname{Fix}\left(h^{2 k}\right)$ is a disjoint union of two simple closed curves, say $F$ and $F^{\prime}$ (see [2]). Let $N=P^{3} /\left\langle h^{2 k}\right\rangle$ and $d: P^{3} \rightarrow N$ be the orbit map. Let $L=d(F)$ and $L^{\prime}=d\left(F^{\prime}\right)$. Then $N$ is homeomorphic to $S^{3}$ and

$$
\pi_{1}\left(N-L-L^{\prime}\right)=Z \oplus Z
$$

(see [2]). Let $f$ be the map of $N$ induced by $h$. Since $f$ is an orientation-reversing map of $N$ (of period $2 k)$ and $d(\operatorname{Fix}(h)) \subset \operatorname{Fix}(f), \operatorname{Fix}(h)$ consists of two points, say $x$ and $\bar{x}$. Let $y=d(x)$ and $\bar{y}=d(\bar{x})$. We may assume that $\{x, \bar{x}\} \subset F^{\prime}$. Then $\operatorname{Fix}(f)=\{y, \bar{y}\} \subset L^{\prime}$. Notice that if $k>1, \operatorname{Fix}\left(f^{2 r}\right)(1 \leqslant$ $\gamma<k)$ is a simple closed curve. Hence Fix $\left(h^{2 r}\right)$ is a simple closed curve, and it is easy to see that $\operatorname{Fix}\left(h^{2 r}\right)=F^{\prime}$ for each $r, r \neq 0(\bmod k)$. Let $M=$ $N /\left\langle f^{2}\right\rangle, g: N \rightarrow M$ be the orbit map (of course, $M=N$ if $k=1$ ). Then $M$ is again homeomorphic to $S^{3}$ (see [3]). Let $T$ be the map of $M$ induced by $f$. Then $T$ is an orientation-reversing involution. Let $J=g(L), J^{\prime}=g\left(L^{\prime}\right), \bar{z}=$ $g(\bar{y})$, and $z=g(y)$. Notice that $\operatorname{Fix}(T)=\{z, \bar{z}\} \subset J^{\prime}$ and $T$ interchanges the two open $\operatorname{arcs} J^{\prime}-\{z, \bar{z}\}$. Since $\pi_{1}\left(N-L-L^{\prime}\right)=Z \oplus Z$, it can be seen that $\pi_{1}\left(M-J-J^{\prime}\right)=Z \oplus Z$ (see [3]).

(3.2) A product structure will be defined on the complement of a regular neighborhood of $J^{\prime}$ in $M$. Take small invariant balls $B$ and $\bar{B}$ in $M-J$, containing $z$ and $\bar{z}$, respectively, such that $B \cap \bar{B}=\varnothing$. Again take a small invariant regular neighborhood of $\operatorname{cl}\left(J^{\prime}-B-\bar{B}\right)$ in $\operatorname{cl}(M-B-\bar{B})-J$ so that it has two components, say $K$ and $K^{\prime}$. Let $\tilde{M}=B \cup \bar{B} \cup K \cup K^{\prime}$. Then $\tilde{M}$ is a regular neighborhood of $J^{\prime}$. Let $\bar{M}=\operatorname{cl}(M-\tilde{M})$. Since

$$
\pi_{1}\left(M-J-J^{\prime}\right)=Z \oplus Z,
$$

it follows from a result of J. Stallings [10] that $\bar{M}$ is homeomorphic to a solid torus $D^{2} \times S^{1}$ and it is also an invariant regular neighborhood of $J$. Parametrize $\bar{M}$ in terms of $A \times I\left(A=S^{1} \times I\right)$ such that

$$
\partial A \times I \approx \operatorname{cl}\left(\partial\left(K \cup K^{\prime}\right)-B-\bar{B}\right), \quad A \times\{0\} \approx \operatorname{cl}\left(\partial B-k-K^{\prime}\right)
$$

and

$$
A \times\{1\} \approx \operatorname{cl}\left(\partial \bar{B}-K-K^{\prime}\right) .
$$

(3.3) Let $h_{i}(i=1,2)$ be an orientation-reversing map of period $4 k$ on $P^{3}$. In connection with $h_{i}$, a symbol such as $q_{i}$ shall be used to represent the same object as $q$ where $q$ is a symbol in (3.1) and (3.2). We will define an equivalence $t$ of $M_{1}$ onto $M_{2}$ such that $T_{2} t=t T_{1}, t\left(J_{1}\right)=J_{2}$, and $t\left(J_{1}^{\prime}\right)=J_{2}^{\prime}$. Since $J$ is isotopic to $S^{1} \times\{1 / 2\} \times\{1 / 2\}$ in $A \times I$ (after parametrizing $\bar{M}$ ), it follows from Lemma 2.2 that there exists an equivalence $\bar{t}$ between $T_{1} \mid \bar{M}_{1}$ and $T_{2} \mid \bar{M}_{2}$ such that $\bar{t}\left(J_{1}\right)=J_{2}, \bar{t}\left(\partial B_{1}-K_{1}-K_{1}^{\prime}\right)=\partial B_{2}-K_{2}-K_{2}^{\prime}$ and $\bar{t}\left(\partial \bar{B}_{1}-K_{1}-K_{1}^{\prime}\right)=\partial \bar{B}_{2}-K_{2}-K_{2}^{\prime}$. Since each $T_{i}$ interchanges $K_{i}$ and $K_{i}^{\prime}$, and $T_{i} \mid B_{i}\left(T_{i} \mid \bar{B}_{i}\right.$ resp.) is essentially the cone over $T_{i} \mid \partial B_{i}\left(T_{i} \mid \partial \bar{B}_{i}\right.$, resp.) (see 
[8]), one can extend $\bar{t}$ to an equivalence $t$ between $T_{1}$ and $T_{2}$ such that $t\left(J_{1}^{\prime}\right)=J_{2}^{\prime}$.

(3.4) Since $\pi_{1}\left(N_{i}-L_{i}^{\prime}\right)=Z$ and $\operatorname{Fix}\left(f_{i}^{2 r}\right)=L_{i}^{\prime}, 1 \leqslant r<k$, there exists a lifting equivalence $s$ between $f_{1}$ and $f_{2}$ such that $g_{2} s=\operatorname{tg} g_{1}$ (see the following diagram). Let $\bar{N}_{i}=g_{i}^{-1}\left(\bar{M}_{i}\right)$ and $\bar{P}_{i}=d_{i}^{-1}\left(\bar{N}_{i}\right)$. Then $\bar{N}_{i}$ and $\bar{P}_{i}$ are invariant regular neighborhoods of $L_{i}$ and $F_{i}$ which are homeomorphic to $D^{2} \times S^{1}$, respectively. Our plan is to find a lifting $r$ of $P^{3}$ such that the following diagram commutes.

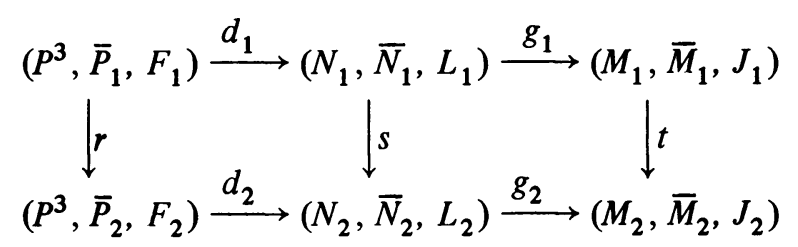

To do this, it is enough to show that $\left(s^{\prime} d_{1}^{\prime}\right)_{\#}\left(\pi_{1}\left(\partial \bar{P}_{1}\right)\right)=d_{2 \#}^{\prime}\left(\pi_{1}\left(\partial \overline{P_{2}}\right)\right)$, where $s^{\prime}: \partial \bar{N}_{1} \rightarrow \partial \bar{N}_{2}$ and $d_{i}^{\prime}: \partial \bar{P}_{i} \rightarrow \partial \bar{N}_{i}$ are the maps such that $s^{\prime}=s \mid \partial \bar{N}_{1}$ and $d_{i}^{\prime}=d_{i} \mid \partial \bar{P}_{i}$. In (3.5) and (3.6) we select some special generators of $\pi_{1}\left(\partial \bar{P}_{i}\right)$ and $\pi_{1}\left(\partial \bar{N}_{i}\right)$. In (3.7) we conclude the proof.

(3.5) Let $q_{i}$ be the orbit map of $T_{i}$ and let $Q_{i}=q_{i}\left(\bar{M}_{i}\right)$. Since $t$ is an equivalence between $T_{1}$ and $T_{2}$, there exists a homeomorphism $\hat{t}$ between the orbit spaces of $T_{1}$ and $T_{2}$ such that $\hat{t q_{1}}=q_{2} t$. Let $q_{i}\left(J_{i}\right)=l_{i}$. It follows from Lemma 2.2 that $Q_{i}$ is a nonorientable disk bundle over $l_{i}$ (see also (3.2)). Therefore one can find an annulus $A_{1}$ in $Q_{1}$ such that $\operatorname{Int}\left(A_{1}\right) \subset \operatorname{Int}\left(Q_{1}\right)$ and $\partial A_{1}$ consists of $l_{1}$ and a simple closed curve $c_{1}$ on $\partial Q_{1}$. Let $A_{2}=\hat{t}\left(A_{1}\right)$. Then $A_{2}$ is an annulus in $Q_{2}$ such that $\operatorname{Int}\left(A_{2}\right) \subset \operatorname{Int}\left(Q_{2}\right)$ and $\partial A_{2}$ consists of $l_{2}$ and a simple closed curve $c_{2}$ on $\partial Q_{2}$.

(3.6) Let $v_{i}=q_{i} \cdot\left(g_{i} \mid \bar{N}_{i}\right)$.Then, since $v_{i}: \bar{N}_{i} \rightarrow Q_{i}$ is the covering projection generated by $\left\langle f_{i} \mid \bar{N}_{i}\right\rangle$, we see that $v_{i}^{-1}\left(A_{i}\right)$ is an annulus in $\bar{N}_{i}$ such that $\partial\left(v_{i}^{-1}\left(A_{i}\right)\right)$ consists of $L_{i}$ and a simple closed curve $v_{i}^{-1}\left(c_{i}\right)$ on $\partial \bar{N}_{i}$, and $\operatorname{Int}\left(v_{i}^{-1}\left(A_{i}\right)\right) \subset \operatorname{Int}\left(\bar{N}_{i}\right)$ (recall that $L_{i}$ is invariant under $f_{i}$ ). On the other hand, there exists a properly embedded disk $D_{i}$ in $\bar{P}_{i}$ such that $D_{i} \cap h_{i}^{2 r}\left(D_{i}\right)=\varnothing$ $(1 \leqslant r<k), h_{i}^{2 k}\left(D_{i}\right)=D_{i}$, and $\partial D_{i}$ does not bound a disk in $\partial \bar{P}_{i}$ (letting $h_{i}^{\prime}=h_{i} \mid \bar{P}_{i}$, then $h_{i}^{\prime 2 k}$ is the only nontrivial element of $\left\langle h_{i}^{\prime}\right\rangle$ with nonempty fixed point set, and one may use the argument in the proof of Lemma 2.8 of [3]). Let $\alpha_{i}$ be the element of $\pi_{1}\left(\partial \bar{P}_{i}\right)$ represented by the path $\partial D_{i}$ and $\beta_{i}$ be another element of $\pi_{1}\left(\partial \bar{P}_{i}\right)$ such that $\alpha_{i}$ and $\beta_{i}$ generate $\pi_{1}\left(\partial \bar{P}_{i}\right)$. Let $\gamma_{i}$ and $\xi_{i}$ be the elements of $\pi_{1}\left(\partial \bar{N}_{i}\right)$ represented by $d_{i}\left(\partial D_{i}\right)$ and $v_{i}^{-1}\left(c_{i}\right)$. Since $d_{i}\left(\partial D_{i}\right)$ bounds a disk in $\vec{N}_{i}$ and $v_{i}^{-1}\left(c_{i}\right)$ is homotopic to the center circle of $\vec{N}_{i}$ $\left(\approx D^{2} \times S^{1}\right)$, we see that $\gamma_{i}$ and $\xi_{i}$ generate $\pi_{1}\left(\partial \bar{N}_{i}\right)$. Since $v_{2} s=\hat{t} v_{1}$, we may assume (by the choice of $c_{i}$ ) that $s_{\#}^{\prime}\left(\xi_{1}\right)=\xi_{2}$ (recall that $\left.s^{\prime}=s \mid \partial \bar{N}_{1}\right)$. Since $d_{i}\left(\partial D_{i}\right)$ bounds a disk in $\bar{N}_{i}$, we may assume that $s_{\#}^{\prime}\left(\gamma_{1}\right)=\gamma_{2}$.

(3.7) We claim that $\bar{v}_{i}^{-1}\left(c_{i}\right)$ is connected, where $\bar{v}_{i}=v_{i} d_{i}$. Suppose that it were disconnected. Recall that $d_{i}^{\prime}=d_{i} \mid \partial \bar{P}_{i}$. Since $d_{i}^{\prime}$ is a double covering projection generated by $h_{i}^{2 k} \mid \partial \bar{P}_{i}$ and $v_{i}^{-1}\left(c_{i}\right)$ is connected, we see that $\bar{v}_{i}^{-1}\left(c_{i}\right)$ 
has two components, say $a_{i}, b_{i}$, and, hence, $h_{i}^{2 k}\left(a_{i}\right)=b_{i}$. Since $\bar{v}_{i} \mid \partial \bar{P}_{i}$ is a covering projection generated by $h_{i} \mid \partial \bar{P}_{i}, a_{i} \cup b_{i}$ is invariant under $h_{i}$ (and $h_{i}^{k}$ ), and either $h_{i}\left(a_{i}\right)=b_{i}$ or $h_{i}\left(a_{i}\right)=a_{i}$ occurs. Therefore we see that $h_{i}^{2 k}\left(a_{i}\right)=a_{i}$, which is a contradiction to the fact that $h_{i}^{2 k}\left(a_{i}\right)=b_{i}$. Hence $\bar{v}^{-1}\left(c_{i}\right)$ is connected. This implies that there is no element $\phi_{i}$ of $\pi_{1}\left(\partial \bar{P}_{i}\right)$ such that $d_{i \#}^{\prime}\left(\varphi_{i}\right)=\xi_{i}$. We may assume (by the choice of $D_{i}$ in $\left.\bar{P}_{i}\right)$ that $d_{i \#}\left(\alpha_{i}\right)=\gamma_{i}^{2}$ and $d_{i \#}^{\prime}\left(\beta_{i}\right)=\gamma^{m_{i}} \xi_{i}$ for some $m_{i}$. Note that $m_{i}$ must be odd (otherwise, $\xi_{i} \in$ $\left.d_{i \#}^{\prime}\left(\pi_{1}\left(\partial \bar{P}_{i}\right)\right)\right)$. Therefore, $d_{i \#}^{\prime}\left(\pi_{1}\left(\partial \bar{P}_{i}\right)\right)$ is generated by $\gamma_{i}^{2}$ and $\gamma_{i} \xi_{i}$. Since $s_{\#}^{\prime}\left(\gamma_{1}^{2}\right)$ $=\gamma_{2}^{2}$ and $s_{\#}^{\prime}\left(\gamma_{1} \xi_{1}\right)=\gamma_{2} \xi_{2}$, we have a lifting $\bar{r}$ of $P^{3}-F_{1}-F_{1}^{\prime}$ to $P^{3}-F_{2}-$ $F_{2}^{\prime}$ such that $d_{2} \bar{r}=s d_{1}$ on $P^{3}-F_{1}-F_{1}^{\prime}$. One may extend $\bar{r}$ to a homeomorphsm $r$ of $P^{3}$ such that $d_{2} r=s d_{1}$. This completes the proof.

\section{Proof of Theorem $C$.}

(4.1) Consider an orientation-reversing map $h$ of period $n$ on $P^{3}$. In $\$ 3$ we have proved the case where $n=4 k$. Now assume that $n=2 k, k$ odd $>1$. Since $h^{k}$ is an involution on $P^{3}, \operatorname{Fix}\left(h^{k}\right)$ is a projective plane $P$ plus an isolated point $x$ (see [6]). Since the period of $h^{2}$ is $k$ (odd), $\operatorname{Fix}\left(h^{2 r}\right)(1 \leqslant r<$ $k$ ) is a simple closed curve (see [3]). Let $F=\operatorname{Fix}\left(h^{2}\right)$. Let $\xi: S^{3} \rightarrow P^{3}$ be the natural projection. Since $\pi_{1}\left(P^{3}\right)=Z_{2}$, it can be seen that $\xi^{-1}(F)$ is a simple closed curve [3], [7]. Since $\pi_{1}\left(P^{3}-F\right)$ is abelian, $\pi_{1}\left(S^{3}-\xi^{-1}(F)\right)=Z$ (see [9]). Therefore, $\pi_{1}\left(P^{3}-F\right)=Z$ (for a proof, see [7]).

(4.2) Since $\pi_{1}\left(P^{3}-F\right)=Z$ and $k$ is odd, the orbit space $M=P^{3} /\left\langle h^{2}\right\rangle$ is homeomorphic to $P^{3}$ (see [3]). Since $P \cup\{x\}$ is invariant under $h$, we see that $h(x)=x$. Since $\operatorname{Fix}(h) \subset F, \operatorname{Fix}(h)$ consists of $x$ and a point $y$ of $F$. Since $h^{2}$ and $h^{k}$ generate the group $\langle h\rangle, F \cap P=\{y\}$. Furthermore, since $h$ interchanges the sides of $P$ in a small neighborhood of $y, F$ meets $P$ at $y$ locally piercingly. Let $g: P^{3} \rightarrow M$ be the orbit map and let $J=g(F)$. Since $\pi_{1}\left(P^{3}-F\right)=Z$, it can be seen that $\pi_{1}(M-J)=Z$ (see [3]). Let $T$ be the involution of $M$ induced by $h$. Since $g h^{k}=T g$, we see that $\operatorname{Fix}(t)=g(P) \cup$ $g(x)$. Let $\bar{P}=g(P)$ and $z=g(x)$. Then $\bar{P}$ is a projective plane and $T$ interchanges the two open arcs of $J-\{z, g(y)\}$. Note that $\bar{P}$ is one-sided in $M$.

(4.3) Triangulate $M$ so that $\operatorname{Fix}(T)$ and $J$ are subcomplexes, and $T$ becomes simplicial. Let $U$ be the simplicial neighborhood of $\bar{P}$ in $M$. Let $B$ be the closed star of $z$ in $M$. We may assume that $B \cap U=\varnothing$. Note that $T \mid B$ is essentially a cone over $T \mid \partial B$ (see [8]). Consider the double covering $\gamma: M^{\prime} \rightarrow$ $M$ obtained from $M$ by cutting along $\bar{P}$. Since $\partial U \approx S^{2}$, we see that $\operatorname{cl}(M-U)$ is homeomorphic to a 3-cell, and $M^{\prime}$ is a 3 -sphere $S^{3}$. Since $\pi_{1}(M-J)=Z$, we see that $\pi_{1}\left(M^{\prime}-J^{\prime}\right)=Z$ where $J^{\prime}=\gamma^{-1}(J)$. Therefore $J^{\prime}$ is unknotted in $M^{\prime}$ (see [9]).

(4.4) Now consider the two orientation-reversing maps $h_{1}$ and $h_{2}$ of $P^{3}$ with period $2 k, k$ odd $>1$. As in (3.3), we use symbols $q_{i}$ in connection with $h_{i}$ $(i=1,2)$ whenever a symbol $q$ has appeared in (4.2). Let

$$
Q_{i}=\operatorname{cl}\left(M_{i}-U_{i}-B_{i}\right)
$$


Let $K_{i}$ be the simplicial neighborhood of $\operatorname{cl}\left(J_{i}-U_{i}-B_{i}\right)$ in $Q_{i}$ such that $K_{i}$ has two components. Since $\gamma_{i}^{-1}\left(U_{i}\right)$ is a product neighborhood $S_{i} \times[-1,1]$ of $\gamma_{i}^{-1}\left(\bar{P}_{i}\right)\left(\approx S^{2}\right)$ such that $S_{i} \times\{0\}=\gamma_{i}^{-1}\left(\bar{P}_{i}\right)$, each component of $\operatorname{cl}\left(M_{i}^{\prime}-\right.$ $\left.\gamma^{-1}\left(U_{i} \cup B_{i}\right)\right)$ is homeomorphic to $S^{2} \times I$ and it is exactly a copy of $Q_{i}$. Therefore, since $J_{i}^{\prime}$ is unknotted in $M_{i}^{\prime}$, a product structure on $\operatorname{cl}\left(Q_{i}-K_{i}\right)$ can be defined in terms of $A \times I\left(A=S^{1} \times I\right)$ such that $\operatorname{cl}\left(\partial B_{i}-K_{i}\right) \approx A \times$ $\{0\}, \operatorname{cl}\left(\partial U_{i}-K_{i}\right) \approx A \times\{1\}$ and $\operatorname{cl}\left(\partial K_{i}-B_{i}-U_{i}\right) \approx S^{1} \times\{0,1\} \times I$. Furthermore the product structure can be chosen so that there exists an involution $f$ on $A$ such that $T_{i}(x, t)=(f(x), t)$ for $(x, t) \in A \times I$ (see $\left.\S 2\right)$. Notice that $T_{i}$ interchanges the two components of $K_{i}$. Therefore, the orbit space of $T_{i} \mid \operatorname{cl}\left(M_{i}-B_{i}-U_{i}\right)$ is homeomorphic to $P^{2} \times I$ (see also [8]) and, letting $G_{i}=M_{i} /\left\langle T_{i}\right\rangle$ and $k_{i}: M_{i} \rightarrow G_{i}$ be the orbit map, there exists a homeomorphism $\alpha$ of $G_{1}$ to $G_{2}$ such that $\left(\alpha k_{1}\right)\left(z_{1}\right)=k_{2}\left(z_{2}\right),\left(\alpha k_{1}\right)\left(J_{1}\right)=$ $k_{2}\left(J_{2}\right)$, and $\left(\alpha k_{1}\right)\left(\bar{P}_{1}\right)=k_{2}\left(\bar{P}_{2}\right)$. Hence there exists an equivalence $\beta$ between $T_{1}$ and $T_{2}$ such that $\beta\left(J_{1}\right)=J_{2}$. Since $\pi_{1}\left(M_{i}-J_{i}\right)=Z$, one may conclude by the lifting theorem that $h_{1}$ and $h_{2}$ are conjugate in the usual way.

\section{REFERENCES} 726.

1. S. Eilenberg, On the problems of topology, Ann. of Math. (2) 50 (1949), 247-260. MR 10,

2. P. K. Kim PL involutions on lens spaces and other 3-manifolds, Proc. Amer. Math. Soc. 44 (1974), 467-473. MR 51 \# 1558.

3. __ Cyclic actions on lens spaces, Trans. Amer. Math. Soc. (to appear).

4. $\longrightarrow$ Periodic homeomorphisms of the 3-sphere and related spaces, Michigan Math. J. 21 (1974), 1-6. MR 50 \#3231.

5. P. K. Kim and J. L. Tollefson, PL involutions of fibered 3-manifolds, Trans. Amer. Math. Soc. (to appear).

6. K. W. Kwun, Scarcity of orientation-reversing PL involutions of lens spaces, Michigan Math. J. 17 (1970), 355-358. MR 43 \#5535.

7. __ Sense preserving PL involutions of some lens spaces, Michigan Math. J. 20 (1973), 73-77.

8. G. R. Livesay, Involutions with two fixed points on the three-sphere, Ann. of Math (2) 78 (1963), 582-593. MR 27 \#5257.

9. C. D. Papakyriakopoulos, On Dehn's Lemma and the asphericity of knots, Ann. of Math. 66 (1957), 1-26.

10. J. Stallings, On fibering certain 3-manifolds, Topology of 3-Manifolds and Related Topics (Proc. Univ. of Georgia Inst., 1961), Prentice-Hall, Englewood Cliffs, N. J., 1962, pp. 92-94. MR 28 \# 1600.

11. F. Waldhausen, On irreducible 3-manifolds which are sufficiently large, Ann. of Math. (2) 87 (1968), 56-88. MR 37 \#146.

12. ___ Über Involutionen der 3-Sphäre, Topology 8 (1969), 81-92. MR 38 \# 5209.

Department of Mathematics, University of Rhode Island, Kingston, Rhode Island 02881

Current address: Department of Mathematics, University of Kansas, Lawrence, Kansas 66045 